\title{
The implementation of
}

a multimedia learning environment for graduate civil engineers

Chris Smith and Peter Jagodzinski

University of Plymouth

This paper examines the underpinning theory, design and implementation of a computer-based Multimedia Learning Environment (MLE) for graduate civil engineers. The MLE brings together multimedia technology and Intelligent Tutoring Systems techniques for the purpose of developing in the graduate engineer the skills and understanding needed to produce initial design proposals for real-world dam spillway design problems.

\section{Introduction}

Underlying our approach to the development of a Multimedia Learning Environment (MLE) is the proposition that conventional learning needs analysis, for Computer-Based Learning does not address the richness of delivery possible with MLEs. We propose that knowledge-engineering techniques (from the field of Artificial Intelligence) can provide a richer understanding of the domain, thus enabling a better MLE to be produced.

The knowledge-engineering approach we have used, outlined in this paper, involved acquiring knowledge from a range of sources, then analysing this knowledge so that individual knowledge items could be categorized according to identified underlying knowledge-representation paradigms which each imply their own, most suitable, presentation style. This knowledge engineering analysis was then used to select an instructional model capable of conveying all the acquired types of knowledge in their paradigmatic styles, and detailed and adaptable enough to be implemented on a computer. The chosen model, the Cognitive Apprenticeship Model (CAM), was explicitly used as the basis for the architecture of the MLE.

This paper is intended to demonstrate the validity of our approach. It concentrates on 
how the acquired knowledge and its representation styles map onto the CAM, the development of an architecture for the MLE, and how the core elements of the CAM can be implemented within the MLE.

\section{Knowledge acquisition}

The developers, who are not civil engineers, were able to compare objectively the relative merits of the three distinct views of the domain knowledge and learning needs proposed by different categories of expert:

(a) The Journeyman. The expert for this part of the process was a post-doctoral research fellow with several years' of recent experience in hydropower design. The first part of the knowledge acquisition process involved this 'Journeyman' explaining an example design problem involving the design of an energy-dissipation device for a dam spillway that was taken from The Design of Small Dams (US Bureau of Reclamation, 1987, 400-402).

(b) The Master. Secondly, we referred to a 'Master', a professor of civil engineering, for confirmation and higher levels of abstraction of knowledge.

(c) The Industry. Thirdly, we were influenced by our discussions with industry and ICE training managers, who identified a number of practical learning needs relating to job performance in the context of the real world.

In addition, a number of research studies and models of the design process were examined (Jeffries et al., 1981, Ullman et al., 1988, and Ball, 1990) to provide confirmation for and a more detailed and comprehensive characterization of the design strategies that seemed to be employed by the Journeyman as he solved the example design problem.

\section{Knowledge representation paradigms underlying the acquired knowledge}

Analysis of the acquired knowledge determined that it could be categorized under three broad classes of representation paradigm:

1. Symbolic (elicited from the Journeyman and confirmed by the Master)

(a) The conceptual and factual knowledge of natural phenomena and engineered structures which can be effectively expressed as equations, graphs or rules to show the relationships between the values involved.

(b) The procedural knowledge of, for example, the steps needed to solve an equation or the steps involved in deriving an equation.

\section{Experiential}

(a) Knowledge and intuitive understanding of complex real-world physical phenomena which can most easily be understood through some physical sensory process, for example the erodability of a stream bed or the buildability of a concrete form. 
(b) Intuitive understanding of values, relationships and abstract theoretical constructs (which may not be observable in the real world) which can be expressed symbolically but which are hard to grasp unless they can be sensorily experienced, for example the force of 1,000 cubic meters of water flowing down a spillway, the kinetic energy of water before and after a hydraulic jump, or the triangle of forces.

Type (a) was disclosed by the Master, and type (b) was stressed by both the Master and the Journeyman.

3. Metacognitive, i.e. the framework within which the symbolic and experiential knowledge is applied in order to accomplish a task.

(a) High-level project structuring metacognitive knowledge, i.e. knowledge that enables the engineer to identify and sequence project activities to provide an activity network for, say, a two-year project. The importance of this type of knowledge was emphasized by industry practitioners.

(b) Lower-level (applicable over a shorter time frame, say one day) knowledge of strategies and heuristics which are needed to be able to apply the symbolic and experiential knowledge so that a small task within the project life-cycle can be accomplished. These strategies were elicited from the Journeyman during his solution of the example design problem, and were confirmed from examination of models of the engineering design process.

\section{Representation styles required by the three categories of knowledge}

Each category of knowledge seems most suited to particular methods of presentation:

Symbolic knowledge (for example mathematical relationships between flow rates and spillway dimensions) needs to be expressed in symbolic terms, i.e. equations or rules. However, much symbolic knowledge can often also be expressed using experiential techniques such as those involving simulation, animation, video and still images, so that it becomes understandable at an intuitive level through sensory experience.

Metacognitive knowledge is often learnt through experience, for example the patterns of procedures and approaches which best suit particular real-world problems. Although it would be possible to teach such strategies and heuristics as rules (i.e. symbolically), in reality a thorough understanding of the subtleties and variations of strategy is acquired only through experience. Such experience can be offered on computer by simulating realworld problem-solving.

Experiential knowledge can thus be seen not as a separate category but as an important alternative perspective on symbolic and metacognitive knowledge which enables these to be understood intuitively as well as formally.

A more detailed account of knowledge elicitation and analysis is given in Jagodzinski et al. (1994). 


\section{A simplified outline of some of the major elements of the CAM}

In order to provide an effective, integrated learning experience, a suitable psychologically recognized, coherent and comprehensive framework was required. After reviewing several approaches, such a framework was found in the Cognitive Apprenticeship Model (CAM) developed by Collins et al. (1989) and Collins (1991). The CAM is an adaptation of the traditional approach of craft apprenticeship for skill-learning into methods for teaching and learning the reasoning and problem-solving cognitive skills of a domain. Its main elements are listed in Table 1.

\section{Content \\ 1.1 Domain knowledge \\ 1.2 Heuristic strategies \\ 1.3 Control strategies \\ 1.4 Learning strategies}

2. Modelling and explaining

\subsection{Modelling of real-world processes}

2.2 Modelling of expert performance

3. Situated learning

4. Coaching

5. Scaffolding

6. Fading

7. Articulation

8. Reflection

9. Exploration

10. 10.1 Increasing complexity

10.2 Increasing diversity

10.3 Global before local skills
Table 1: Elements of the Cognitive Apprenticeship Model (CAM)

The knowledge content of the CAM approach is defined in the first two of its elements, as shown in Figure 1 which illustrates way in which the acquired knowledge and its representation styles map onto the CAM. Domain knowledge (1.1) corresponds with conventional declarative text-book knowledge, and 1.2 to 2.2 , various aspects of procedural knowledge including problem solving strategies and modelling real-world performance. Together, these two aspects of the CAM are able to comprehend the type of knowledge representation required by the domain of dam spillway design.

The instructional process of the CAM (elements 3 to 10 in Table 1) conveys metacognitive knowledge and improves understanding of the symbolic and its related experiential knowledge by placing all teaching within the context of real-world problem solving. This is accomplished by having the tutor first model the whole solution to a problem (element 2.2 in Table 1). Then work begins on a similar problem with the tutor carrying out some parts of the problem and the learner attempting the other parts of it. Work continues on similar 


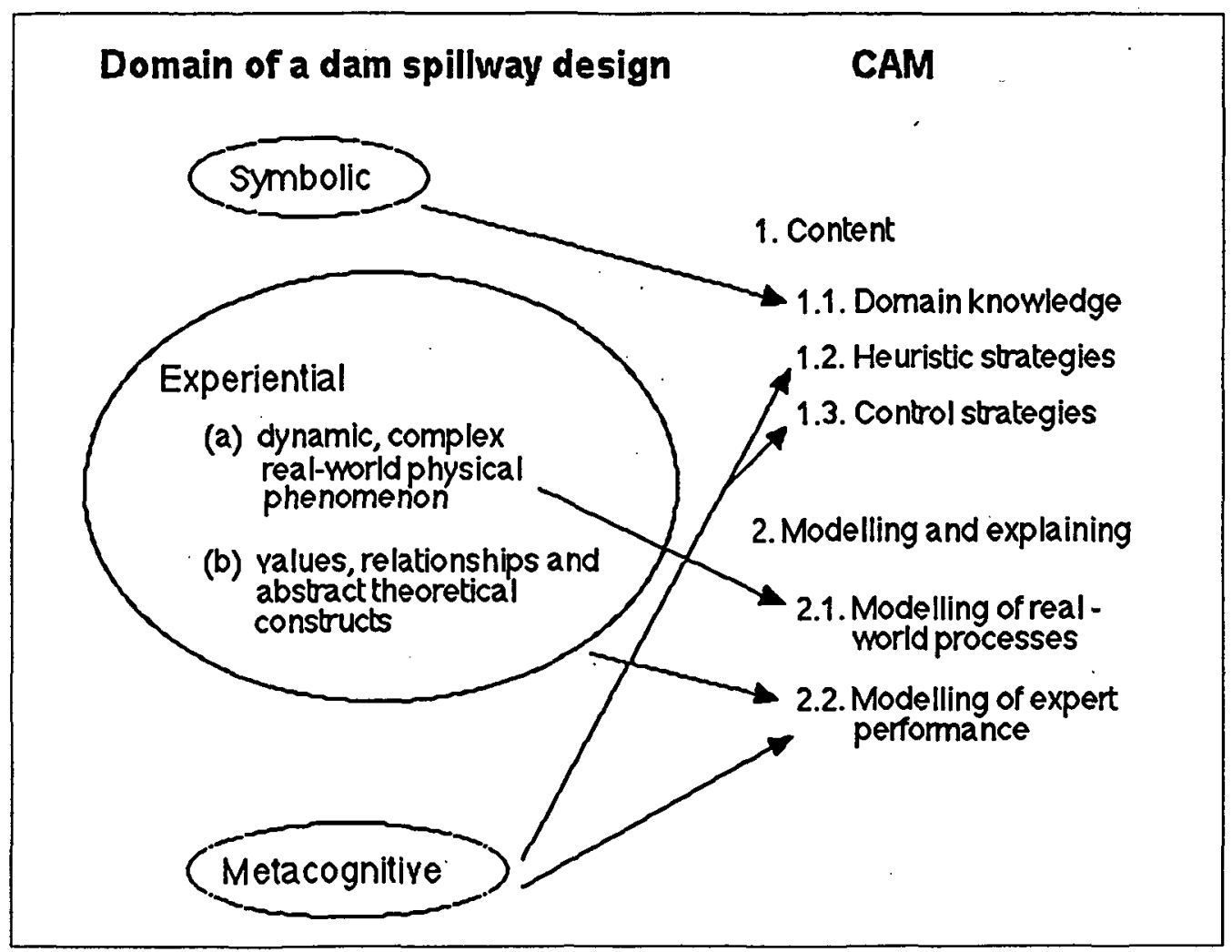

Figure 1: How the knowledge representation paradigms of the domain of dam spillway design map onto the types of knowledge recognized by the CAM

new problems with the learner taking on as much of the new problem as possible, as soon as possible, until he or she can comfortably solve a whole problem on his or her own.

Having the tutor carry out parts of the problem is termed 'scaffolding' (element 5 in Table 1) within the CAM, and having learners take on more and more of the problem by themselves is known as 'fading' the scaffolding (element 6 in Table 1). The CAM offers guidance on the provision and removal of scaffolding. Initially, support should be offered to learners to relieve them of the lower-level skills needed to perform a complex task so that they first examine, practise and learn the higher-level reasoning skills necessary to carry out the task. They thus develop a conceptual map of the overall activity (which is also gained from modelling) before they go on to develop specific skills. This is known as teaching 'global before local' skills (element 10.3 in Table 1). The CAM also suggests that coaching (element 4 in Table 1) should be provided. This involves monitoring learners as they work, and offering hints, scaffolding, feedback, modelling, reminders, and new tasks, all of which are intended to move their performance closer to the performance of an expert. 
The way in which these core CAM elements of modelling, coaching, scaffolding and fading can be provided by the MLE will be discussed below. A detailed examination of the other important aspects of the CAM is given in Brown and Newman (1989) and Collins (1991).

\section{An architecture for the MLE}

Figure 2 provides a schematic overview of the components of the MLE.

The 'coach' first models the expert solution of a problem. The learner is shown the solution sequentially, a page at a time. Pages already seen can be re-examined by being

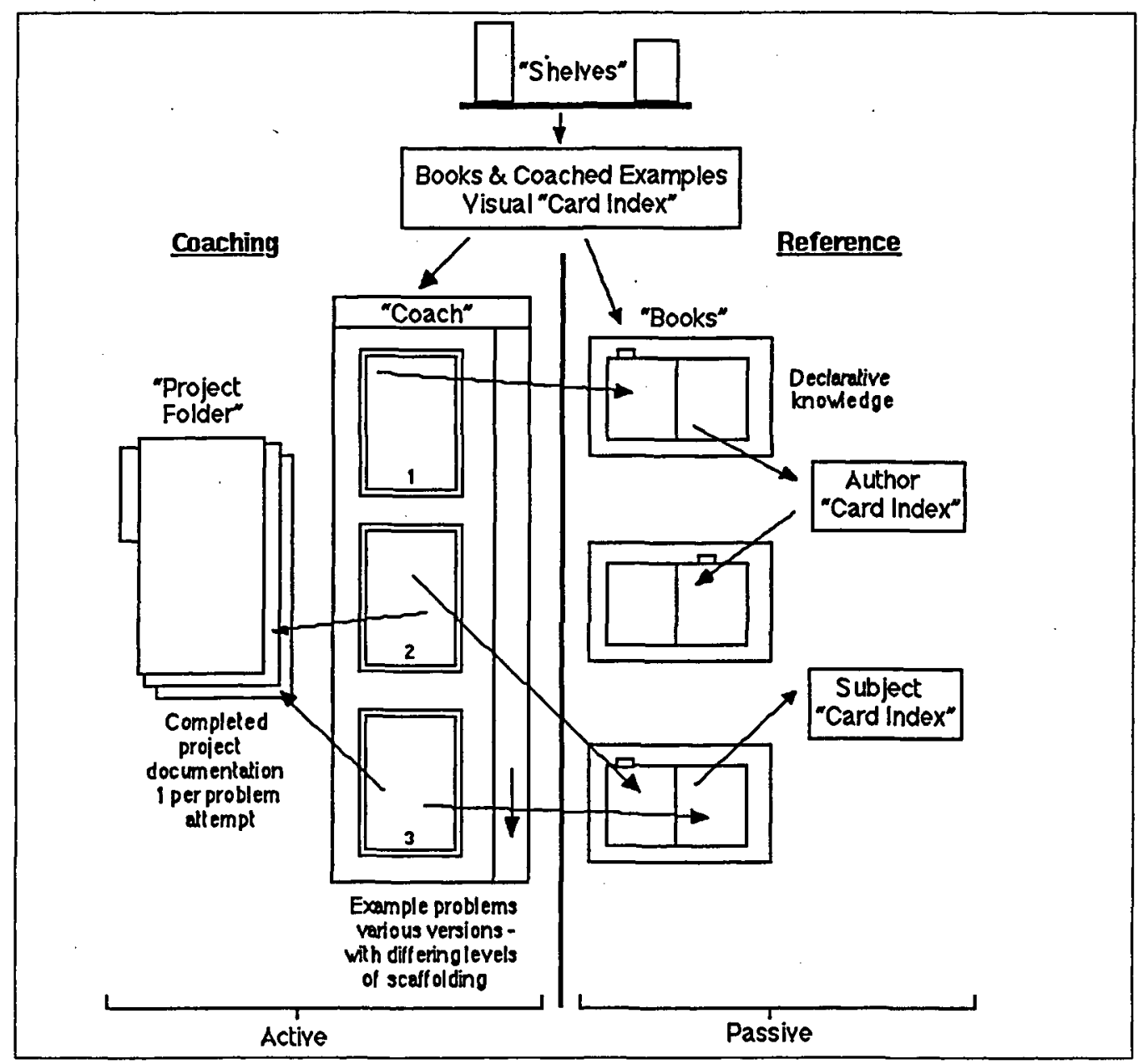

Figure 2: MLE orchitecture the text 
scrolled through. After being shown how to solve a problem, the learner can then sequentially work his or her way through a similar type of problem but one which uses different values. The system will support the learner by alternately demonstrating the solution to part of the problem and then asking him or her to attempt part of the problem, and so on. This is done according to the 'global before local' sequencing of skills.

As a case study problem is worked through, values are filled in on examples of project documentation held within the 'project folder'. This provides students with a correct model of the appropriate ways of recording their findings. 'Books' of reference material are referred to in the 'coach' to provide background understanding for learners and to help them complete their parts of the task. The 'books' are structured and contain organized multimedia explanations and demonstrations of symbolic and related experiential knowledge which can be used to solve many different types of problem (see Figure 3 for examples). 'Books' are referenced within 'card indexes' and the coached examples themselves are also initially taken from an 'index'. All 'books', 'coached examples' and 'indexes' are available on a number of 'shelves'.

After completing a section, learners ask for it to be evaluated. If they have done badly, they can be helped. For example, the system can demonstrate the solution to that section, and levels of demonstration with varying detail can also be shown. If learners reach an impasse, they can, again, be given similar help. How well a learner does overall on a runthrough determines how much of the scaffolding is faded for the next coached run-through of a similar problem with different values.

\section{The Coach}

The following is a simplified outline of the underlying architecture and mechanisms needed to implement the CAM's core elements of modelling, coaching, scaffolding and fading.

\section{Modelling}

Each of the tasks in Figure 4, which shows the decomposition of a typical problem into individual steps, would be represented as a node within the system. Each node has a set of 'system demonstration' pages and a set of 'student work-through' pages associated with it. The system demonstration pages illustrate how an expert would solve the task, and the student work-through pages invite students to solve the task for themselves. Initially, the expert's solution to the problem, using a particular set of values, is modelled. This is accomplished by using a stack to deliver the system demonstration pages for each node, in turn, i.e. $a, b, c, d, e$ and $f$.

\section{Scaffolding and fading}

Each leaf node in Figure 4 (b, d, e and $\mathrm{f}$ ) also has an evaluation procedure, a student score and a difficulty rating and sequence ranking (see Figure 5). A task's difficulty rating is a measure of how complex and time-consuming a task is to complete. The task sequence rankings are used in the process of allocating the next task to be attempted by the student. 


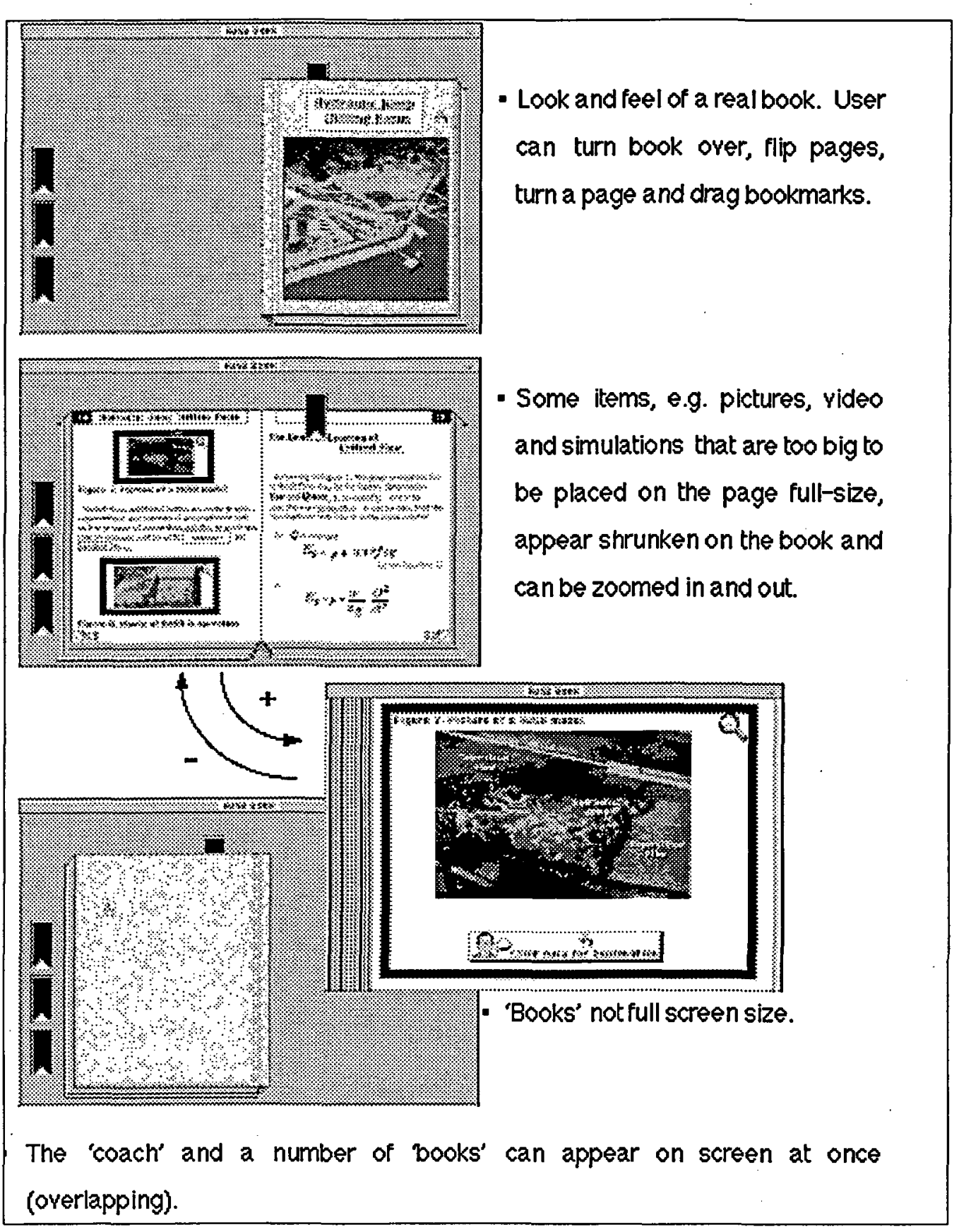

Figure 3: Examples of screens from a book 
Figure 4: A generic design problem decomposed into a number of tasks

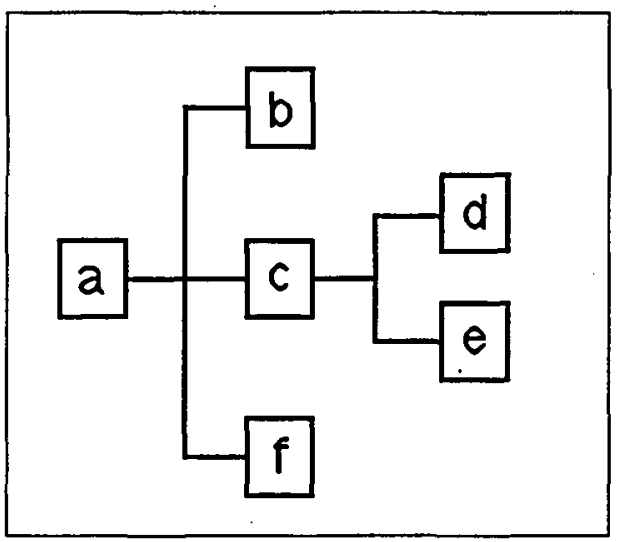

On a 'scaffolded run-through', the same design problem is used but with different values, so different choices may have to be made. For example, in one of our civil engineering problems, different types of spillway stilling basin may need to be considered.

When 'scaffolding', the system will demonstrate some parts of the problem, and the learner will attempt other parts of the problem. The basic method for allocating tasks to the learner uses the students scores, difficulty ratings and sequence rankings:

- Leaf nodes are ordered according to their sequence rankings and difficulty ratings: $f(S R=1, D R=40), e(S R=2, D R=10), d(S R=2, D R=20), b(S R=3, D R=30)$.

- Say $50 \%$ of the given problem (in terms of difficulty) is to be allocated to the learner on

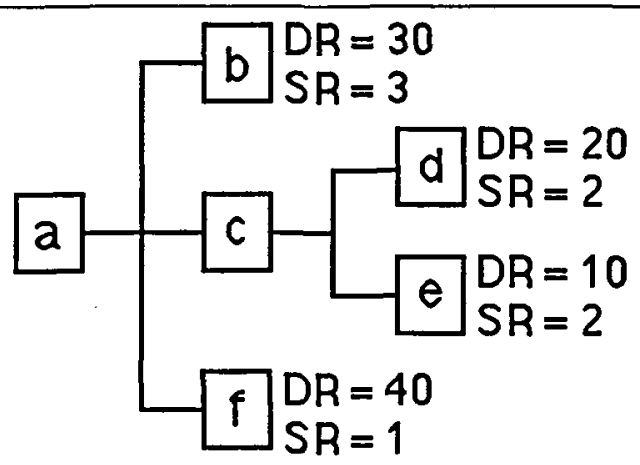

DR - Difficulty Rating, SR - Sequence Ranking

A 'real' problem would include many more task nodes.

Figure 5: Design problem nodes with example difficulty ratings and sequence rankings 
the next run-through (the higher a learner's overall score on a run-through, the more of the problem he or she will have to attempt on the next run-through).

- $50 \%$ of the total difficulty of the whole problem is calculated, i.e. 50 (in this case). So, a number of tasks with a difficulty totalling 50 (or as close to it as possible without exceeding it) will have their node settings set to show student work-through pages. Thus, during the run-through, at these points, the student will be asked to attempt those tasks on his or her own.

- The first tasks whose difficulty ratings total 50 , or as near as possible to 50 , are allocated to the student according to his or her sequence rankings. So, on the next runthrough, nodes $f(S R=1, D R=40)$ and $e(S R=2, D R=10)$ will be allocated to the student to attempt.

- The sequence rankings are intended to deliver the CAM's 'global before local' sequencing because the centrally important guiding tasks and decisions will be given higher sequence rankings in order to ensure that students will practise them earlier.

\section{Coaching}

Each section attempted by a student is evaluated. If the student does badly on a section, he or she can be shown the system demonstration pages for the node concerned, its child nodes and so on (varying demonstration/help pages can be given depending on the errors made). If a student is unable to continue on a section, again he or she can be shown the system demonstration pages for the node concerned and its child nodes (and, again, varying demonstration/hint pages can be given depending on the experience of the student, for example how much of the problem he or she has been allocated).

\section{Conclusion}

Our use of a knowledge-engineering approach to the development of multimedia learning environments has brought four benefits:

\section{Recognition of the need for a range of domain expertise.}

2. A richer perception of the learning domain - three categories of knowledge have been recognized, and the importance of experiential knowledge in particular has been an unexpected revelation.

3. A set of paradigms for the representation of knowledge in the architecture of the MLE.

4. A basis for selecting an instructional model for the architecture of the MLE which is capable of integrating and delivering the different types of knowledge in their most appropriate representation styles.

The CAM model has been of enormous value in providing us with a clear and detailed rationale for the way in which material is presented to learners, for the modelling of their performance and for the interaction between these two elements.

The premise of this research was that careful attention to design can unlock the potential 
of multimedia as a learning tool. We believe that we have shown that the approaches we have used are capable of facilitating a penetrating understanding of a learning domain, and consequently a rich design which situates the learning environment in the context of realistic problem-solving.

\section{References}

Ball, L.J. (1990), Cognitive Processes in Engineering Design, Ph.D. thesis, University of Plymouth.

Collins, A., Brown, J.S. and Newman, S.E. (1989), 'Cognitive apprenticeship: teaching the crafts of reading, writing and mathematics' in Resnick, B. (ed), Essays in Honour of Robert Glaser, Hillsdale NJ, Lawrence Erlbaum.

Collins, A., (1991), 'Cognitive apprenticeship and instructional technology' in Idol, L. and Jones, B.F. (eds), Educational Values and Cognitive Instruction: Implications for Reform, Hillsdale NJ, Lawrence Erlbaum.

Jagodzinski, P., Parmee, I. and Smith, C. (1994), 'The use of multiple paradigms for the representation of knowledge in a multimedia learning environment for engineering design', paper submitted to the International Journal of Human-Computer Studies.

Jeffries, R., Turner, A.A., Polson, P.G. and Atwood, M.E. (1981), 'The processes involved in designing software' in Anderson, J.R. (ed), Cognitive Skills and their Acquisition, Hillsdale NJ, Lawrence Erlbaum.

Ullman, D.G., Dietterich, T.G. and Stauffer, L.A. (1988), 'A model of the mechanical design process based on empirical data', $A I E D A M 2,1,33-52$.

United States Department of the Interior, Bureau of Reclamation (1987), The Design of Small Dams (third edition), Denver CO, United States Government Printing Office. 